\title{
PREDICCIÓN DEL FLOW DISPOSICIONAL SEGÚN EL CLIMA MOTIVACIONAL Y EL TRATO GENERADO POR EL PROFE- SOR EN CLASE DE EDUCACIÓN FÍSICA
}

\author{
Juan Antonio Moreno Murcia* \\ Eduardo Cervelló Gimeno** \\ Celestina Martínez Galindo*** \\ Néstor Alonso Villodre ${ }^{* \star *}$ \\ Universidad de Murcia* \\ Universidad Miguel Hernéndez (Elche)** \\ Unidad de Investigación en Educacalón Fisica y Deportes****
}

\section{RESUMEN}

El objetivo de este trabajo fue estudiar la relación entre el clima motivacional percibido y la percepción de trato de igualdad o discriminación generado por el profesor en el aula sobre el flow disposicional experimentado por el discente en clase de educación fisica, con una muestra compuesta por 1103 alumnos de entre 13 y 14 años. Para ello, se realizó un análisis factorial confirmatorio (CFA), análisis de correlación entre las variables estudiadas y análisis de ecuaciones estructurales. Los resultados mostraron que el clima motivacional que implicaba a la tarea predecia positivamente la percepción de igualdad de trato y el flow disposicional, mientras que predecla negativamente la percepción de discriminación. Por el contrario, el clima motivacional que implicaba al ego predecia positivamente la percepción de discriminación y de forma negativa la percepción de igualdad de trato y el flow disposicional. A diferencia de la 
mayoría de estudios ya existentes que han analizado el flow disposicional en entornos deportivos $y$, sobre todo competitivos, la presente investigación lo aborda desde un contexto educativo, al considerar que en este tipo de ambientes el sujeto también puede presentar estados psicológicos óptimos de ejecución.

Palabras clave: EDUCACIÓN FÍSICA, CLIMA MOTIVACIONAL, IGUALDAD DE TRATO-DISCRIMINACION, FLOW.

\section{SUMMARY}

The objective of this work was to study the relationships between the perception of motivational climate, perception of equality treatment or discrimination generated by the teacher in the classroom on the dispositional flow experienced by the students in physical education classes. The sample was 1103 students between 13 and 14 years. A confirmatory factor analysis (CFA), correlation analysis and analysis of structural equations modeling were employed. The results showed that the perception of a task-involving climate predicted positively the perception of equality treatment and dispositional flow, while it predicted negatively the perception of discrimination. The perception of an ego-involving climate predicted positively the perception of discrimination and in a negative way the perception of equality treatment and the dispositional flow. Contrary to most of studies already existent that have analyzed dispositional flow in sport environments (mainly competitive) the present investigation approaches it from an educational context, when considering that in this type of atmosphere the subject can also exhibit a good psychological state.

Key words: PHYSICAL EDUCATION, MOTIVATIONAL CLIMATE, DISCRIMINATION-EQUALITY TREATMENT, FLOW. 


\section{INTRODUCCIÓN}

Junto al entorno social y familiar en el que se encuentra inmerso el sujeto, el ámbito educativo constituye uno de los ambientes más importantes para formar y educar a los alumnos. En concreto, dentro de este ámbito, el entorno creado por las clases de educación fisica constituye un marco ideal para permitir el desarrollo tanto físico, como psíquico y social de todo el alumnado. Por ello, consideramos fundamental conocer y comprender los diferentes procesos de ensef̂anza-aprendizaje que tienen lugar en el aula de educación física con el fin de mejorar la educación tanto académica como personal de todos los discente ya que, según diferentes investigaciones relacionadas con la temática, la adquisición de experiencias de aprendizaje positivas en la adolescencia relacionadas con la actividad física, repercutirán en el estilo de vida adquindo en la etapa adulta $y$, consecuentemente, en los niveles de práctica físico-deportiva en tiempo de ocio y recreación.

Al respecto, un amplio cuerpo de investigaciones han confirmado los indudables beneficios fisicos y psicológicos que aporta la actividad física practicada de forma regular, produciendo un impacto importante en el bienestar y en la calidad de vida de quienes la practican. En línea con estas afirmaciones, la evidencia sería un incremento paulatino de participación en actividades fisico-deportivas, sin embargo, contrario a esta afirmación lógica, existe un tendente declive en el disfrute de tales actividades a lo largo de la vida (Owen y Bauman, 1992), muy fundamentalmente, en la etapa adolescente y, más concretamente, en el sexo femenino.

Por ello, un amplio cuerpo de investigación se ha centrado en determinar las razones que justifican las marcadas actitudes negativas que mantienen las adolescentes hacia la actividad físico-deportiva y, más concretamente, hacia la asignatura de educación física, alegando como causa fundamental la falta de motivación expenimentada durante la clase como consecuencia, entre otras, del trato desigual recibido por su profesor en el aula. Así, en ocasiones, el propio docente de educación física puede contribuir con su actuación a potenciar la discriminación de sexo en la clase. Según Griffin (1989), los educadores físicos no tiene generalmente la intención de tratar a los 
alumnos de forma desigual, sin embargo, hay docentes que de forma no intencional discriminan a algunos alumnos mediante el uso de estereotipos racistas o sexistas. De tal manera que, unas veces la intencionalidad y en otras ocasiones el escaso nivel de experiencia de algunos profesores hacen que los alumnos en clase de educación física no reciban una verdadera experiencia de equidad en función de su sexo (Del Villar, 1996).

Al respecto, esta discriminación puede ser transmitida a través de diferentes elementos integrados en el curriculum de educación fisica. En esta línea, Askew y Ross (1988), Jiménez, Ramos, y Cervelló (2002) y Williams (1993), señalan como elementos transmisores de discriminación del curriculum oculto, aquellos elementos propios del comportamiento del profesorado, aquellos elementos propios del uso de determinados elementos metodológicos y aquellos elementos integrados en los propios discentes. En este sentido, la labor del profesor va a resultar fundamental para minimizar y erradicar las posibles situaciones generadoras de desigualdad entre los alumnos a través de su conducta, así como de su metodología empleada ya que, aspectos tales como el interés, la satisfacción, el comportamiento de disciplina y el grado de implicación del alumno son variables mediatizadas por la percepción que el alumno tiene del clima generado por su profesor (Clark, 1971; Dauer y Pangrazi, 1989; Harrinson y Blakemore, 1989).

Por ello, diferentes estudios (Papaioannou, 1995, 1998; Cervelló, Jiménez, Del Villar, Ramos, y Santos-Rosa, 2004; Cervelló y Jiménez, 2001; Jiménez, 2004) han analizado la influencia de los factores ambientales de la teoría de metas en la percepción del trato generado por el profesor en el aula, encontrando que el clima que implica a la tarea favorece la implicación del alumno en las actividades propuestas, genera mayores conductas disciplinadas y mayores sentimientos de igualdad de trato, mientras que el clima que implica al ego, favorece el sedentarismo, la indisciplina y la percepción de discriminación.

Como podemos observar, ha existido una significativa progresión cuantitativa y cualitativa de los estudios realizados en el ámbito de la psicología de la actividad física y el deporte centrados en determinar, analizar y minimizar el efecto de las variables que pueden afectar al rendimiento personal y académico de los alumnos intentando para 
ello determinar las consecuencias de las experiencias negativas durante la práctica de educación física, apoyándose, en la mayoría de los casos, en los diferentes tipos de motivación que guian el comportamiento de los alumnos en el aula.

Sin embargo, ha sido menos extensa la línea de investigación centrada en determinar los procesos cognitivos que contribuyen a la mejora del rendimiento y de la satisfacción en la actividad física y el deporte, obviando muchos de ellos, el análisis de las experiencias subjetivas vividas por los sujetos durante la práctica físico-deportiva (García Calvo, Cervelló, y Santos-Rosa, 2006). Experiencias que se corresponderían con un estado psicológico óptimo para la realización de una actividad mostrando una disposición mental propicia para llevar a cabo la tarea planteada y que Csikszentmihalyi (1975) denominó "Estado de Flow», abarcando dicho término 9 dimensiones que vendrían a definirlo: equilibrio entre habilidad y reto, combinación/unión de la acción y el pensamiento, claridad de objetivos, feedback claro y sin ambigüedades, concentración sobre la tarea que se está realizando, sentimiento de control, perdida de cohibición o de autoconciencia, transformación en la percepción del tiempo y experiencia autotélica (Csikszentmihalyi, 1990, 1997; Jackson, 1996).

Atendiendo a diferentes estudios, existe una relación directa entre el clima generado por el adulto significativo y el estado psicológico alcanzado por el sujeto. De tal manera que, en entomos deportivos Cervelló, Fenoll, Jiménez, García-Calvo, y Santos-Rosa (2001), Moreno, Cervello, y González-Cutre (2006) han encontrado que el flow disposicional es predicho tanto por la orientación al ego y a la tarea como por la percepción de un clima motivacional que implica tanto a la tarea como al ego. Resultados similares a los hallados por Alonso (2006) en un contexto de educación física. Una nota común de todos estos estudios es que, si bien tanto el ego como la tarea predicen el flow disposicional, es esta última dimensión la que lo hace en mayor medida.

Como se puede apreciar, este constructo ha sido estudiado en su totalidad en entornos de actividad física y deporte, siendo muy pocos los que analizan dicho constructo en ámbitos de educación física. Consideramos que, por ser un entorno que guarda estrecha relación con el ámbito deportivo, los alumnos en clase de educación física pueden llegar a experimentar estados psicológicos óptimos en la 
realización de las diferentes tareas propuestas por el profesor, pudiéndose ver afectada esta relación por el trato generado por el docente en el aula.

Considerando la importancia de ampliar las líneas de investigación existente y atendiendo a las prospectivas de investigación señaladas por García Calvo et al. (2006) en torno a la necesidad de seguir investigando la relación existente entre el estado de flow y diversas variables psicológicas para abrir y mejorar el estudio y comprensión de dicho estado psicológico óptimo, y con ello, mejorar el proceso de enseñanzaaprendizaje que tienen lugar en el aula, nos planteamos como principal objetivo en nuestra investigación: estudiar la relación existente entre el clima motivacional imperante en el aula, la percepción del trato de igualdaddiscriminación generado por el profesor y el flow disposicional alcanzado por los discentes. Así, según las aportaciones teóricas revisadas se hipotetiza que el clima motivacional que implica a la tarea predecirá positivamente la percepción de igualdad de trato y negativamente la percepción de discriminación, mientras que el clima motivacional que implica al ego predecirá positivamente la percepción de discriminación y negativamente la percepción de igualdad de trato. La percepción de igualdad de trato predecirá positivamente el flow disposicional, mientras que la percepción de discriminación lo hará de forma negativa.

\section{MÉTODO}

\section{Muestra}

La muestra del estudio estuvo compuesta por un total de 1103 sujetos (606 hombres y 497 mujeres) cuyas edades oscilaron entre los 13 y 14 años. La totalidad de la muestra perteneció a una población de estudiantes de primer ciclo ( $2^{\circ}$ curso) de Educación Secundaria Obligatoria de diferentes centros escolares públicos y privados de la provincia de Alicante (España).

\section{Instrumentos}

Cuestionario de percepción de igualdad-discriminación en educación fisica (CPIDEF). Utilizamos el Cuestionario de Percepción 
de Igualdad-Discriminación en educación fisica (Cervelló et al., 2004) para medir la percepción de igualdad-discriminación trasmitida por el profesor en el aula. El cuestionario constaba de 19 ítems, 10 de los cuales perteneclan a conductas de «igualdad de trato" (i.e. «Emplea las mismas normas para las alumnas que para los alumnos») y los otros 9 items correspondian a conductas de "discriminación» (i.e. «Nos propone actividades distintas a las chicas que a los chicos»). Las respuestas a dicho cuestionario fueron cerradas y respondian a una escala tipo Likert que iba de 0 , valor que correspondla a totalmente en desacuerdo, a 10 que indicaba que el alumno estaba totalmente de acuerdo con lo que se le planteaba. Dicho inventario mostró unos resultados alpha de .84 para la subescala igualdad de trato y de .72 para la subescala discriminación. Recientemente, Alonso, Martínez Galindo, y Moreno (2006) han confirmado su estructura factorial obteniendo unos adecuados indices de ajuste, $(\div 2(35, N=1103)=438.06, p<.000, \div 21=3.71, \mathrm{CFI}=$ $.94, \mathrm{NFI}=.92, \mathrm{TLI}=.93, \mathrm{SRMR}=.04, \mathrm{RMSEA}=.05$ ).

Cuestionario del Clima Motivacional en las clases de Educación Física (CMEF). Utilizamos el instrumento de medición del clima motivacional en las clases de Educación Fisica (CMEF) validado por Alonso (2006) para medir la percepción de los discentes del clima motivacional que transmitía su profesor en las clases de educación fisica. Dicho cuestionario constaba de 24 ítems, 12 de los cuales correspondian a conductas del profesor que inducian al «clima motivacional que implica a la tarea» (i.e. "Mi profesor/a nos evalúa por hacer las cosas cada vez mejor") y otros 12 , correspondian a conductas del profesor que induclan al «clima motivacional que implica al ego" (i.e. "Mi profesor/a me corrige comparándome con mis compañeros/as»). Las respuestas a dicho cuestionario fueron cerradas y respondian a una escala tipo Likert que iba de 0 (totalmente en desacuerdo) a 10 (totalmente de acuerdo). El inventario mostró unos resultados alpha de .82 para la subescala clima motivacional que implica a la tarea y de .77 para la subescala clima motivacional que implica al ego.

Cuestionario del flow disposicional (DFS). Utilizamos la versión en español de la Escala de Flow Disposicional (DFS) de Jackson (2000) traducida por Santos-Rosa (2003). El inventario constaba de 36 ítems 
cuyas respuestas fueron cerradas y respondian a una escala tipo Likert que oscilaba de 0 (totalmente en desacuerdo) a 10 (totalmente de acuerdo). Dicha escala tiene un factor principal (Flow disposicional) (i.e. «Hice los gestos correctos sin pensar, de forma automática») y nueve factores secundarios (equilibrio entre habilidad y reto, combinación/unión de la acción y el pensamiento, claridad de objetivos, feed-back claro y sin ambigüedades, concentración sobre la tarea que se está realizando, sentimiento de control, pérdida de cohibición o de autoconciencia, transformación en la percepción del tiempo y experiencia autotélica) basados en las nueve características que explican un estado de rendimiento óptimo (Csikszentmihalyi, 1990 . 1993). Dicho cuestionario mostró valores alpha de .92 para el factor flow (que recoge sus nueve dimensiones posibles).

\section{Procedimiento}

Para llevar a cabo el estudio y conseguir los objetivos propuestos, en primer lugar nos pusimos en contacto con los padres/tutores de los sujetos menores de edad que componen la muestra de estudio, a los que se les informó del objetivo de la investigación y se les solicitó su autorización para que su hijos o tutorados pudieran participar en la misma. Seguidamente, se procedió a la administración de los cuestionarios bajo la supervisión del investigador principal, con el fin de solventar toda duda que pudiera surgir en la comprensión tanto de los ítems que componian los cuestionarios como de las instrucciones establecidas al inicio de la administración de los mismos. El tiempo aproximado para completar los cuestionarios fue de 20-25 minutos. La participación fue voluntaria y la recogida de los instrumentos se realizó de forma individual para verificar que ningún ítem quedara sin contestar.

\section{Análisis de datos}

Para confirmar la estructura factorial de las escalas empleadas en el estudio, así como testar la validez de los factores compartiendo un método común que tiende a aumentar las correlaciones entre las variables medidas a través de los constructos (Perugiri y Conner, 
2000), se realizó un análisis factorial confirmatorio (modelo de medición). En segundo lugar, para determinar la relación existente entre las variables estudiadas, se llevó a cabo un análisis de correlación entre el clima motivacional percibido, la percepción del trato generado por el profesor en el aula (igualdad o discriminación) y el flow disposicional. En último lugar, se llevó a cabo un análisis del modelo de ecuaciones estructurales propuesto con el que se pretendia testar simultáneamente el modelo estructural y el de medición, permitiéndonos, con ello, centrarnos en las correlaciones conceptuales existentes entre los factores latentes y las variables de medición.

\section{RESULTADOS}

\section{Análisis del modelo de medición}

Para poder realizar el análisis del modelo de medición y testar el modelo de ecuaciones estructurales (SEM) que se presentará más adelante, uno de los aspectos que se tuvo en cuenta fue reducir el número de indicadores de los factores latentes, debido a que el tamaño muestral no es particularmente amplio en comparación con el amplio número de variables que componen el modelo (Marsh, Richard, Johnson, Roche, y Tremayne, 1994; Ntoumanis, 2001; Sarrazín, Vallerand, Guillet, Pelletier, y Cury, 2002; Vallerand, 1997). Así, uno de los primeros pasos que realizamos para mantener unos grados de libertad razonables consistió en agrupar de forma aleatoria los ítems de cada constructo en dos grupos aleatorios (Bagozzi y Heatherton, 1994; Bentler, 1980; Byrne, 1994; MacCallum y Austin, 2000), debido a que, según Marsh et al. (1994), esta agrupación de ítems resulta más fiable, permite distribuirlos de forma más normal y la proporción entre el número de variables medidas en el modelo y el número de sujetos que componen la muestra de estudio queda reducida a la mitad.

De esta manera, por ejemplo, en la escala CPIDEF, el factor «igualdad de trato» quedó compuesto por dos grupos aleatorios de cinco ítems cada uno, y el factor «discriminación» por dos grupos aleatorios de cinco y cuatro ítems, respectivamente. Así con cada 
uno de los factores que componen cada una de las escalas administradas a la muestra de estudio.

Una vez divididos los items que componen cada uno de los factores latentes en dos grupos aleatorios, se realizb un análisis factorial confirmatorio (CFA) basándonos en las 10 medidas observadas y los 5 constructos latentes que correlacionan libremente (Anderson y Gerbing, 1988) (Figura 1).

Para comprobar la validez del modelo de medición se utilizó el programa estadistico Amos en su versión 5.0, y se consideraron una serie de coeficientes fit o indices de bondad de ajuste. Asi, basándonos en las aportaciones de diferentes autores (Bentler, 1990; Bollen y Long, 1993; McDonald y Marsh, 1990; Mulaik et al., 1989), los indices fit ó indices de bondad de ajuste que se consideraron para evaluar la bondad del modelo de medición fueron: $\div 2, \div 2 / \mathrm{g}$.l., RMSEA (Root Mean Square Error of Aproximation), SRMR (Standardized Root Mean Square Residual) y los indices incrementales (CFI, NFI y TLI). Estos indices de bondad de ajuste son considerados aceptables cuando el $+2 / g . l$. es inferior a 5, los indices incrementales (CFI, NFI y TLI) son superiores a .90 y los índices de error (RMSEA y SRMR) son inferiores a .05 (Ntoumanis, 2001).

Como se observa en la figura 1, el modelo de medición proporcionó unos indices fit adecuados, $\div 2(25, N=1103)=87.36, p<.000, \div 21$ g.l. $=3.49, \mathrm{CFI}=.98, \mathrm{NFI}=.98, \mathrm{TLI}=.98, \mathrm{SRMR}=.01, \mathrm{RMSEA}=$ $.04)$, resultando todos los parámetros significativos $(t>2)$. Además, se examinó la validez discriminante del modelo, teniendo en cuenta que la correlación entre las variables latentes, atenuadas por el error de medición (+/- 2 veces el error de medición), fuera inferior a 1.0. Así, los resultados obtenidos del análisis factorial confirmatorio revelaron que el modelo de medición no mide mal.

\section{Medias, desviaciones típicas y análisis de correlación}

En este apartado se presentan los estadísticos descriptivos, medias y desviaciones típicas, y las correlaciones obtenidas entre el clima motivacional percibido, la percepción del trato de igualdad o discriminación transmitido por el docente y el flow disposicional (Tabla 1). Los datos revelaron una puntuación mayor en la percepción de un 


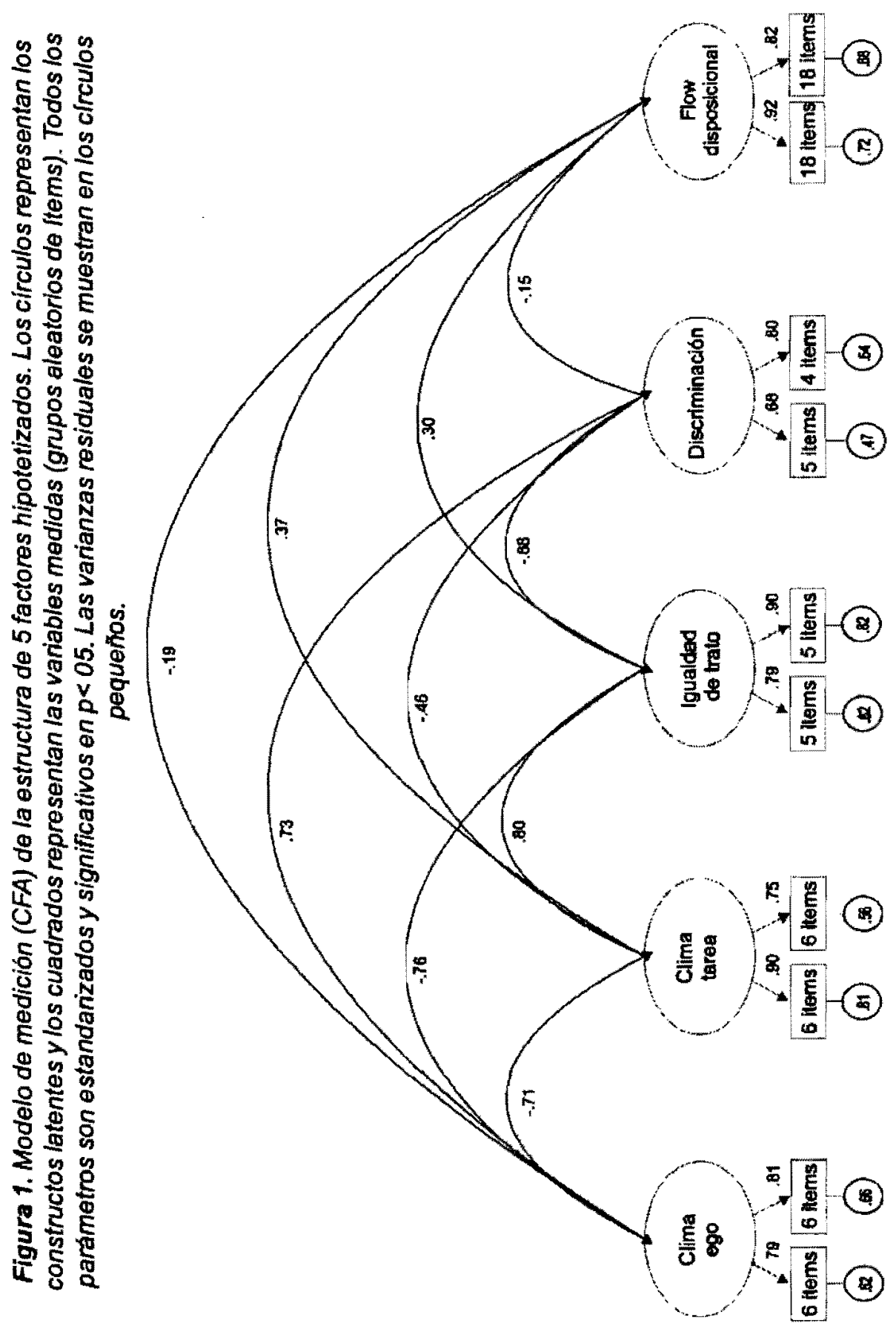


clima motivacional que implicaba a la tarea $(M=6.07)$ que en la percepción de un clima motivacional que implicaba al ego $(M=2.96)$. Así como una mayor puntuación en la percepción de un trato de igualdad $(M=7.70)$ que de discriminación $(M=3.43)$. La puntuación media en el flow disposicional fue de $M=6.49$.

Los datos obtenidos del análisis de correlación revelaron que el clima motivacional que implicaba a la tarea se relacionaba de forma positiva y significativa con la percepción de igualdad de trato $(r=.65, p<.01)$ y el flow disposicional $(r=.32, p<.01)$, mientras que lo hacía de forma regativa y significativa con el clima motivacional que implicaba al ego ( $r$ $=-.56, p<.01)$ y la percepción de discriminación $(r=-.35, p<.01)$. Por su parte, el clima motivacional que implicaba al ego se relacionaba de forma positiva y significativa con la percepción de discriminación ( $r=$ $.54, p<.01)$ y de forma negativa y significativa con la percepción de igualdad de trato $(r=-.60, p<.01)$, la percepción de un clima motivacional que implicaba a la tarea $(r=-.56, p<.01)$ y el flow disposicional $(r=-.17$, $p<.01)$, aunque esta última correlación obtuvo un valor bajo $(<.30)$.

Tabla 1. Media, desviación típica y correlaciones de todas las variables.

\begin{tabular}{lcccc}
\hline Factores & $M$ & $D T$ & 1 & 2 \\
\hline 1. Clima tarea & 6.07 & 1.90 & - & $-.56^{* *}$ \\
2. Clima ego & 2.96 & 1.77 & - & - \\
3. Igualdad de trato & 7.70 & 2.06 & - & - \\
4. Discriminación & 3.43 & 2.29 & - & - \\
5. Flow disposicional & 6.49 & 1.58 & - & - \\
\hline
\end{tabular}

$\star p<.05 ; * * p<.01$

\section{Análisis de modelos de ecuaciones estructurales}

Con el objeto de analizar las relaciones e interacciones existentes entre las variables pertenecientes al modelo que se plantea, se ha utilizado el Modelo de Ecuaciones Estructurales (SEM: Structural Equation Modeling) a través del programa estadístico AMOS 5.0 (Figura 
2). Para comprobar la bondad o semejanza del modelo teórico propuesto con los datos empíricos existentes, se han tenido en cuenta una serie de índices fit o indices de bondad de ajuste, descritos en el apartado anterior. AsI, los datos obtenidos fueron: $(\div 2 \mathrm{2}(28, N=1103)=166.83$, $\mathrm{p}<.000, \div 2 \mathrm{~g} . \mathrm{I}$ = $=5.95, \mathrm{CFI}=.97, \mathrm{NFI}=.97, \mathrm{TLI}=.96, \mathrm{SRMR}=.03$, RMSEA $=.06$ ). Estos datos se ajustan a los parámetros establecidos, por lo que podemos considerar aceptado el modelo propuesto. De igual forma, se examinó la contribución de cada uno de los factores a la predicción de otras variables a través de los pesos de regresión estandanizados. El valor «t» asociado a cada peso se tomó como una medida para comprobar la contribución, de modo que, se consideraron significativos valores superiores a 1.96 .

Como podemos observar en la figura 2, los climas motivacionales percibidos por los discentes (clima motivacional que implica a la tarea y clima motivacional que implica al ego) aparecen como variables exógenas, es decir, no son predichas por ninguna variable, mientras que el resto de variables que componen el modelo actúan como variables endógenas, o lo que es lo mismo, predicen y a la vez son predichas por otras variables. De esta manera, el presente modelo ofrece los climas motivacionales como predictoras de la percepción de los discentes de la igualdad de trato o discriminación mostrada por el profesor en el aula y del flow disposicional.

El modelo propuesto relacionó negativamente los climas motivacionales entre sí, ya que, como defendió Duda (2001), ambos climas motivacionales (clima motivacional que implica a la tarea y clima motivacional que implica al ego) se encuentran negativa y moderadamente relacionados $(\beta=-.72)$, sugiriendo que los dos constructos pueden ser percibidos de forma independiente. Del mismo modo, se obtuvo que el clima motivacional que implicaba a la tarea predecia positivamente la percepción de igualdad de trato $(\beta=.45)$ y negativamente la percepción de discriminación $(\beta=-.50)$, mientras que el clima motivacional que implicaba al ego predecia positivamente la percepción de discriminación $(\beta=.93)$ y negativamente la percepción de igualdad de trato $(\beta=-.20)$. De igual forma, obtuvimos que la percepción de igualdad de trato predecía positivamente el flow disposicional $(\beta=.41)$, mientras que la percepción de discriminación lo hacía de forma negativa $(\beta=-.13)$. 

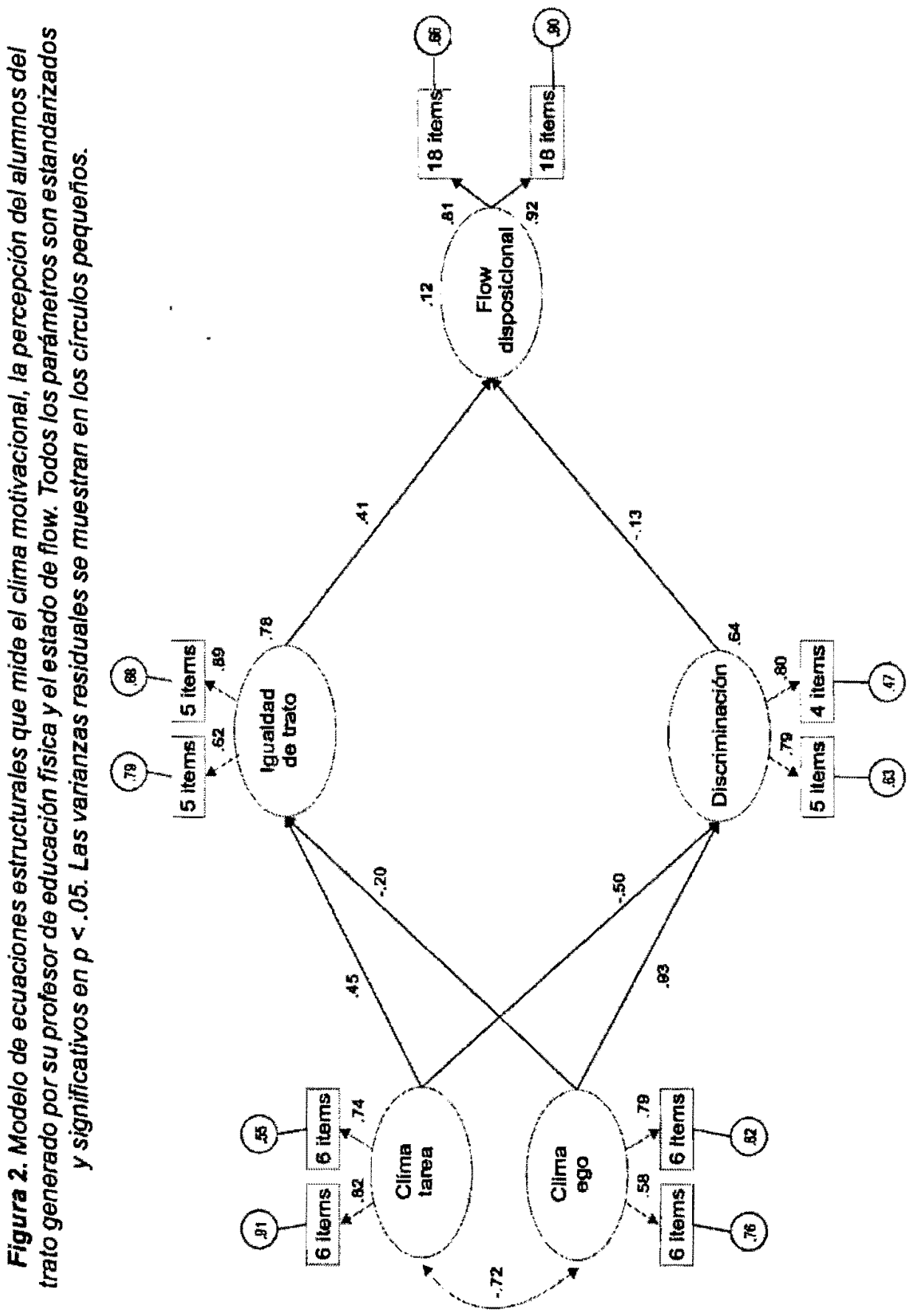


\section{DIscusión}

El objetivo de la investigación ha sido estudiar la relación existente entre diferentes variables, tales como, la percepción del clima transmitido por el profesor en el aula, la percepción del trato de igualdaddiscriminación generado por el docente y el flow disposicional, motivados por la necesidad de ampliar las líneas de investigación entorno al flow disposicional en contextos educativos $y$, asi, poder mejorar los procesos de enseñanza-aprendizaje que tienen lugar en el aula. La originalidad de este estudio radica en el hecho de estudiar el flow disposicional dentro de un contexto educativo, a diferencia de la mayoria de estudios ya existentes que se han centrado en un contexto de actividad física y deporte y, además, en la mayoría de los casos, dentro de un contexto competitivo.

Los resultados obtenidos mostraron, tal y como se hipotetizó, que el clima motivacional que implicaba a la tarea predecía positivamente la percepción de igualdad de trato y negativamente la percepción de discriminación, mientras que el clima motivacional que implicaba al ego predecla positivamente la percepción de discriminación y negativamente la percepción de igualdad de trato. De igual forma, obtuvimos que la percepción de igualdad de trato predecia positivamente el flow disposicional, mientras que la discriminación lo hacia de forma negativa. El modelo propuesto obtuvo unos adecuados índices de ajuste, por lo que podemos determinar que no mide mal. De igual forma, previo a la realización del modelo, llevamos a cabo un análisis factorial confirmatorio (modelo de medición) a través del cual confirmamos la estructura factorial de las escalas empleadas en el estudio al obtener unos adecuados indices de bondad de ajuste.

Así, los resultados encontrados coinciden con los encontrados por Cervelló et al. (2004), Jiménez (2004), Martínez Galindo (2006) y Papaioannou $(1995,1998)$, quienes, añadiendo la variable orientación disposicional, mostraron una correlación positiva entre la orientación a la tarea y la percepción de un clima motivacional que implica a la tarea con la percepción de igualdad de trato y entre la onientación al ego y la percepción de un clima motivacional que implica al ego con la percepción de discriminación. Al respecto, Papaioannou $(1995,1998)$ argumentó que un indicador crítico de la igualdad de trato hacia chicos 
y chicas consistía en la docencia bajo un clima que implicaba a la tarea, de tal manera que este tipo de clima correlacionaba positivamente con los profesores que no mantenian diferencia de trato entre chicos y chicas, referente a la focalización del aprendizaje, motivación y ánimo proporcionado.

En relación con el flow disposicional, no encontramos estudios que relacionen este estado con la percepción del trato generado por el profesor en el aula. Así, justificamos los datos encontrados siguiendo los resultados de las investigaciones señaladas, así como los postulados de la teoría de las metas de logro (Nicholls, 1989), según los cuales, los elementos que componen el clima motivacional hacen referencia al modo en el que se utilizan los sistemas de recompensas, la manera en la que se diseñan las prácticas, la forma en la que se agrupan a los sujetos y la manera en la que las figuras de autoridad evalúán el rendimiento (Escartl y Brustad, 2000; Escartl y Gutiérrez, 2001). En este sentido, el profesor que enfatice la percepción de un clima motivacional que implique al ego otorgará recompensas al que consiga la meta final, sin importar el aprendizaje, diseñará prácticas basadas en la competición, agrupará a los alumnos atendiendo a su nivel o habilidad, de tal manera que, en un grupo se situarán los habilidosos y en otro los menos habilidosos o utorpes", y evaluará el rendimiento basándose en la comparación pública entre iguales. En este ambiente, los alumnos percibirán un trato desigual o discriminatorio por parte del profesor, debido a que tan solo los alumnos aventajados disfrutarán de un mayor y mejor trato por parte del docente, siendo, generalmente, las alumnas las que experimenten en mayor medida este trato discriminatorio por presentar menor habilidad en los deportes competitivos, debido, en cierta medida, a que este tipo de actividad no se corresponde con sus intereses ni actitudes hacia la práctica de actividad fisica-deportiva y que, sin embargo, predominan en las clases de educación física, constituyendo un elemento de discriminación. Será lógico que en este tipo de ambientes el discente carezca de un estado psicológico óptimo debido a la situación de estrés y ansiedad que le puede llegar a producir el estar en un ambiente en el que se sienta en continua competición con sus iguales. Consecuentemente, carecerá del control sobre la ejecución a realizar y percibirá que su habilidad no se adecua al reto a conseguir, bien por 
ser superior lo que le generará sentimientos de aburrimiento y desmotivación, bien por ser inferior lo que le provocará sentimientos de fracaso y abandono.

Por el contrario, el profesor que genere un clima motivacional que implique a la tarea, dará recompensas al alumno que mejore su habilidad, que se esfuerce, que aprenda y que muestre interés, independientemente de la consecución final del resultado, diseñará prácticas desafiantes basadas en el reto personal y agrupará a los alumnos aleatoriamente, independientemente de su nivel de habilidad o capacidad. De tal manera que, permitirá la cooperación entre los alumnos y evaluará atendiendo a la superación personal, al esfuerzo aplicado en la tarea y al grado de interés, diversión, satisfacción y motivación manifestada durante la tarea. Bajo este ambiente, los alumnos apreciarán mayor igualdad de trato por parte del profesor, debido a que percibirán que la consecución del éxito dependerá de él mismo y no de su nivel de habilidad ni de su comparación con el resto de compañeros, no importando, por lo tanto, el sexo del alumno. Bajo este tipo de ambientes y bajo esta percepción del trato generado por el profesor, el alumno adecuará los objetivos y las metas a conseguir, consiguiendo con ello mayor control sobre la situación y, consecuentemente, un mayor equilibrio entre su habilidad y el reto a conseguir, permitiéndole todo ello una ejecución más automática, debido a su mayor implicación en la tarea a realizar. De igual forma, en relación con lo citado, si el discente tiene claro cuáles son los objetivos a perseguir y como conseguirlos, es más probable que también perciba claramente si lo que está realizando se adecua a esos objetivos o por el contrario tiene que cambiar su forma de actuar para poder lograrlo (feed-back claro y sin ambigüedades), consiguiendo con ello tener control completo sobre la actividad, $y$, lo más importante, disfrutar con la misma. En definitiva, alcanzará un estado psicológico óptimo.

Por el contrario, diferentes estudios han obtenido resultado contrarios a lo citado señalando que tanto en contextos que implican al ego como a la tarea se puede conseguir un flow disposicional (Moreno, Alonso, Martínez Galindo, y Cervello, 2005; Santos-Rosa, 2003). Justificamos la diferencia existente debido al hecho de que estos estudios fueron realizados en contextos deportivos y, en algunos 
de los casos competitivos, por lo que resulta lógico que para conseguir un estado óptimo de ejecución, se necesite de un clima motivacional que implique al ego en el que el entrenador potencie la necesidad de superar a los demás, por cuanto que, en definitiva, el deporte, y más aún el deporte competitivo, se rige por la máxima de ganar a los demás. Por el contranio, en entornos educativos, esta máxima no debe de primar ya que el docente de educación fisica debe potenciar la mejora, el progreso y el aprendizaje por encima de la comparación, superación entre iguales y competición. $Y$, en el caso de que en ocasiones se genere dicho clima, el profesor deberá tener en cuenta la percepción de competencia de los discentes, ya que un alumno que perciba baja competencia, eludirá competir con los demás debido a que considerara que el fracaso será inminente, generando este sentimiento, entre otras cosas, abandono de la actividad fisico-deportiva a realizar.

Sugerimos la necesidad de corroborar o refutar los resultados encontrados en la presente investigación con muestras de diferentes poblaciones y culturas, asI como de seguir investigando los diferentes factores (motivacionales, conductuales, actitudinales, etc.) que pueden facilitar la consecución de dicho estado, ya que conocerlos permitirá establecer una serie de premisas para mejorar la intervención, de manera que se incremente el rendimiento personal y académico de los discentes, a la vez que se proporcionen experiencias gratificantes que garanticen el compromiso con la actividad flsica, en sus diferentes ámbitos.

\section{REFERENCIAS BIBLIOGRÁFICAS}

Alonso, N. (2006). Motivacion, comportamientos de discipiina, trato de igualdad y flow en estudlantes de educación fis/ca. Tesis Doctoral. Murcia: Universidad de Murcia.

Alonso, N., Martínez Galindo, C., y Moreno, J. A. (2006). Análisis factorial confirmatorio del cuestionario de percepción de igualdaddiscriminación en educación fisica (CPIDEF) en alumnos adolescentes de educación física. En M. A. González, J. A. Sánchez, y A. Areces (Eds.), IV Congreso de la Asociación Española de Ciencias del Deporte (pp. 693-696). A Coruña: Xunta de Galicia. Anderson, J. C., y Gerbin, D. W. (1988). Structural equation modeling 
in practice: a review y recommended two-step approach. Psychological Bulletin, 103, 411-423.

Askew, S., y Ross, C. (1988). Boys don't cry. Boys and sexism in education. Milton Keynes: Open University Press.

Bagozzi, R. P., y Heaherton, T. F. (1994). A general approach to representing multifaceted personality constructs: apllication to state self-esteem. Structural Equation Modeling, 133, 35-67.

Bentler, P. M. (1980). Multivariate análisis with latent variables: causal modeling. Annual Review of Psychology, 31, 419-456.

Bentler, P. M. (1990). Comparative fit indexes in structural models. Psychological Bulletin, 107, 238-246.

Bollen, D. A., y Long, J. S. (1993). Testing Structural Equation Models. Sage: Newbury Park, CA.

Byrne, B. (1994). Structural Equation Modeling with EQS and EQS/ Windows. Sage: Thousy Oks, CA.

Cervello, E. M., Fenoll, A. N., Jiménez, R., García-Calvo, T., y SantosRosa, F. J. (2001). Un estudio piloto de los antecedentes disposicionales y contextuales relacionados con el estado de flow en competición. Comunicación presentada en el II Congreso de Ciencias de la Actividad Fisica y el Deporte, Valencia.

Cervellb, E. M., y Jiménez, R. (2001). Un estudio correlacional entre la orientación motivacional, el clima motivacional percibido, la coeducación y los comportamientos de disciplina en las clases de Educación Fisica. Comunicación presentada en el IV Congreso Intemacional sobre la enseñanza de la Educación Física y el Deporte Escolar, Santander.

Cervell6, E., Jiménez, R., Del Villar, F., Ramos, L., y Santos-Rosa, F. J. (2004). Goal orientations, motivational climate, equality, and discipline of spanish physical education students. Perceptual and Motor Skills, 99, 271-283.

Clark, G. H. (1971). A process of obtaining information and attitudes of children regarding elementary school. Unpublished doctoral thesis, Colombia University

Csikszentmihalyi, M. (1975). Beyond boredom and anxiety. San Francisco: Jossey-Bass.

Csikszentmihalyi, M. (1990). Flow: The psychology of optimal experience. New York: Harper \& Row. 
Csikszentmihalyi, M. (1993). The evolving self. New York: Harper Collins.

Csikszentmihalyi, M. (1997). Finding Flow: The psychology of engagement with everyday life. New York: Basic Books.

Dauer, V., y Pangrazi, R. (1989). Dynamic physical education for elementary school children. New York: Macmillan Publishing Company.

Del Villar, F. (1996). La investigación en la enseñanza de la Educación Flsica. En F. Del Villar (Ed.), La evolución del pensamiento docente durante las prácticas didácticas. Un estudio de casos en Educación Fisica (pp. 203-231). Cáceres: Servicio de Publicaciones, Universidad de Extremadura.

Duda, J. L. (2001). Achievement goal research in sport: Pushing the boundaries and clarifying some misunderstyings. En G.C. Roberts (Ed), Advances in motivation in sport and exercise (pp. 129-182). Champaign, IL: Human Kinetics.

Escartl, A., y Brustad, R. (2000). El estudio de la motivación deportiva desde la perspectiva de la teoría de metas. Comunicación presentada en el I Congreso Hispano-Portugués de Psicologia, Santiago de Compostela. España.

Escarti, A., y Gutierrez, M. (2001). Influence of the motivational climate in Physical Education on the intention to practice physical activity or sport. European Journal of Sport Science, 1 (4), http:/l humankinetics.com /products/journal/index.cfm?id=EJSS

García Calvo, T., Cervello, E., y Santos-Rosa, F. J. (2006). Flow y deporte. En E. J. Garcés de los Fayos, A. Olmedilla, y P. Jara (Eds.), Psicologla y deporte. Murcia: Diego Marín.

Griffin, P. S. (1989). Gender as a socializing agent in Physical Education. En T.J. Templin y P.G. Schempp (Eds.), Leaming to teach: Socialization into Physical Education (pp. 219-233). Indianapolis, IN: Benchmark Press.

Harrinson, J., y Blakemore, C. (1989). Instructional strategies for secondary school physical eduation. Dubuque, IA: William C. Brown Publishers.

Jackson, S. A. (1996). Toward a conceptual understanding of the flow experience in elite athletes. Research Quarterly for Exercise and Sport, 67, 76-90. 
Jackson, S. A. (2000). The Dispositional Flow Scale-2 and the Flow State Scale-2. En J. Maltby, C.A. Lewis, y A. Hill (Eds.), Comissioned reviews of 250 psychologycal tests (pp. 50-52, 61-63). Lampter, U.K: Edwin Mellen.

Jiménez, R. (2004). Motivación, trato de igualdad, comportamientos de disciplina y estilos de vida saludables en estudiantes de Educación Fisica en Secundaria. Tesis Doctoral. Cáceres: Universidad de Extremadura.

Jiménez, R., Ramos, L., y Cervello, E. (2002). Análisis de la coeducación en las clases de Educación Física. Propuestas didácticas para una intervención no sexista en el contexto educativo. Habilidad Motriz, 18, 39-47.

MacCallum, R. C., y Austin, J. T. (2000). Applications of structural equation modeling in psychological research. Annual Review of Psychology, 51, 201-226.

Marsh, H. W., Richard, G. E., Johnson, S., Roche, L., y Tremayne, P. (1994). Physical self-decription questionnaire: Psychometric properties and a nultitrait-multimethod analysis of relations to existing instruments. Journal of Sport and Exercise Psychology, $16,270-305$.

Martínez Galindo, M. (2006). Motivación, coeducación y disciplina en estudiantes de educacion fisica. Tesis Doctoral. Murcia: Universidad de Murcia.

McDonald, R. P., y Marsh, H. W. (1990). Choosing a multivariate model: noncentrality and goodness of fit. Psychological Bulletin, 107, 247-255. Moreno, J. A., Alonso, N., Martínez Galindo, C., y Cervelló, E. (2005). Motivación, disciplina, coeducación y estado de flow en Educación Física: diferencias según la satisfacción, la práctica deportiva y la frecuencia de práctica. Cuadernos de Psicologia del Deporte, 5, 225-243.

Moreno, J. A., Cervelló, E., y González-Cutre, D. (en prensa). The achievement goal and self-determination theories as predictors of dispositional flow in young athletes. International Journal of Sport Psychology.

Mulaik, S. A., James, L. R., Van Astine, J., Bennett, N., Lind, S., y Stilwell, C. D. (1989). Evaluation of goodness-of-fit indices for structural equation models. Psychological Bulletin, 105, 430-445. 
Nicholls, J. G. (1989). The competitive ethos and democratic education. Cambridge, MA: Harvard University Press.

Ntoumanis, N. (2001). A self-determination approach to the understying of motivation in physical education. British Joumal of Educational Psychology, 71, 225-242.

Owen, N., y Bauman, A. (1992). The descriptive epidemiology of physical inactivity in adult Australians. International Journal of Epidemiology, 21, 305-310.

Papaioannou, A. (1995). Differential perceptual and motivational patterns when different goals are adopted. Journal of Sport and Exercise Psychology, 17, 18-34.

Papioannou, A. (1998). Students' perceptions of the physical education class environment for boys and girls and the perceived motivational climate. Research Quarterly for Exercise and Sport, 69, 267-275.

Perugini, M., y Conner, M. (2000). Predicting and understying behavioral volitions: The interplay between goals and behaviours. European Journal of Social Psychology, 30, 705-731.

Santos-Rosa, F. J. (2003). Motivación, ansiedad y flow en jóvenes tenistas. Tesis doctoral. Cáceres: Universidad de Extremadura.

Sarrazin, P., Vallerand, R., Guillet, E., Pelletier, L., y Cury, F. (2002). Motivation and dropout in female hyballers: a 21-month prospective study. European Journal of Social Psychology, 32, 395-418.

Vallerand, R. J. (1997). Toward a hierarchical model of intrinsic and extrinsic motivation. En M.P. Zanna (Ed.), Advances in experimental social psychology (pp. 271-360). Academic Press: New York.

Williams, V. (1993). Who cares about girls? Equality, Physical Education and the primary school child. En J. Evans (Ed.), Equality, Education and Physical Education (pp. 125-138). London: The Falmer Press. 